\title{
$N$-acetylglucosaminyltransferase IVa promotes invasion of choriocarcinoma
}

\author{
KIMIHIRO NISHINO $^{1}$, EIKO YAMAMOTO ${ }^{1,2}$, KAORU NIIMI $^{1}$, \\ YOKO SEKIYA $^{1}$, YORIKO YAMASHITA ${ }^{3}$ and FUMITAKA KIKKAWA ${ }^{1}$
}

\author{
Departments of ${ }^{1}$ Obstetrics and Gynecology, and ${ }^{2}$ Healthcare Administration, Nagoya University \\ Graduate School of Medicine, Nagoya 466-8550; ${ }^{3}$ Department of Experimental Pathology and Tumor Biology, \\ Nagoya City University Graduate School of Medical Sciences, Nagoya 467-8601, Japan
}

Received February 26, 2017; Accepted May 2, 2017

DOI: 10.3892/or.2017.5661

\begin{abstract}
Gestational trophoblastic neoplasia (GTN) results from the malignant transformation of placental trophoblasts which secrete human chorionic gonadotropin (hCG) as do normal placenta or hydatidiform mole. $\mathrm{N}$-acetylglucosaminyltransferase IV (GnT-IV) is a glycosyltransferase which catalyses the formation of $\beta 1,4 \mathrm{GlcNAc}$ branches on the mannose core of $N$-glycans. Previous studies reported that $\beta 1,4 \mathrm{GlcNAc}$ branches on hCG were detected in GTN but not in normal pregnancy or hydatidiform mole. The aim of the present study was to understand the role of GnT-IVa in choriocarcinoma and find the target proteins for GnT-IVa glycosylation which contribute to the malignancy of choriocarcinoma. Immunohistochemistry showed that Griffonia simplicifolia lectin-II staining and GnT-IVa staining were intense in trophoblastic cells of invasive mole and choriocarcinoma. We established a choriocarcinoma cell line with GnT-IVa overexpression (Jar-GnT4a), and examined its malignant potential and target proteins for GnT-IVa glycosylation. GnT-IVa overexpression increased the cell migration and invasion (2.5- and 1.4-fold) as well as the ability to adhere to the extracellular matrix (ECM) components, including fibronectin and collagen type I and IV. The tumour formation potential of Jar-GnT4a in mice was significantly higher than that of control $(\mathrm{P}=0.0407)$, and the cumulative survival rate of mice with Jar-GnT4a was relatively lower than those with control. Immunoprecipitation studies showed that $\beta 1,4 \mathrm{GlcNAc}$ branches of $\mathrm{N}$-glycans on integrin $\beta 1$ in choriocarcinoma cells were increased by GnT-IVa overexpression. Nano-LC/MS/MS analysis
\end{abstract}

Correspondence to: Dr Eiko Yamamoto, Department of Health care Administration, Nagoya University Graduate School of Medicine, 65 Tsurumai-cho, Showa-ku, Nagoya 466-8550, Japan E-mail: yamaeiko@med.nagoya-u.ac.jp

Key words: choriocarcinoma, integrin $\beta 1$, invasion, $2, \mathrm{~N}$-acetyl glucosaminyltransferase IVa, lysosome-associated membrane glycoprotein suggested that lysosome-associated membrane glycoprotein 2 (LAMP-2) was a target protein for glycosylation by GnT-IVa. The increase in $\beta 1,4 \mathrm{GlcNAc}$ branches on LAMP- 2 by GnT-IVa overexpression was confirmed by lectin blot analysis using whole cell lysate and conditioned medium. Our results suggest that highly branched $\mathrm{N}$-glycans generated by the action of GnT-IVa are present in trophoblastic cells of GTN in proportion to GnT-IVa expression level, and that GnT-IVa may contribute to the malignancy of choriocarcinoma by promoting cell adhesion, migration and invasion through glycosylation of integrin $\beta 1$ and LAMP-2.

\section{Introduction}

Gestational trophoblastic disease (GTD) is a group of diseases that has its origins in trophoblasts. The common characteristics of GTD are abnormal proliferation of atypical trophoblasts and secretion of human chorionic gonadotropin (hCG). GTD consists of hydatidiform mole, invasive mole, choriocarcinoma, placental site trophoblastic tumour (PSTT) and epithelioid trophoblastic tumour (ETT) (1). Hydatidiform mole is an abnormal pregnancy resulting from impaired fertilization. Other GTDs are grouped under gestational trophoblastic neoplasia (GTN) which has the potential for local invasion and metastasis $(1,2)$. Common GTNs are invasive mole and choriocarcinoma which respond to chemotherapy. However, $\sim 10 \%$ of choriocarcinoma patients have a poor prognosis, particularly when they have metastasis other than pulmonary metastasis $(3,4)$. hCG is a unique reliable marker for monitoring GTD $(2,4)$.

Structural changes of branched $\mathrm{N}$-glycans on certain glycoproteins are mediated by a group of enzymes named $N$-acetylglucosaminyltransferases (GnTs) which add $N$-acetylglucosamine (GlcNAc) branch antennae to the mannose core of $\mathrm{N}$-glycans. These modifications of $\mathrm{N}$-glycans alter the biological functions of glycoproteins and eventually, the properties of cells containing these glycoproteins (5). $N$-glycan modifications mediated by GnTs greatly influence the invasion and migration abilities of cancer cells $(6,7)$. $N$-acetylglucosaminyltransferase IV (GnT-IV) catalyses the formation of $\beta 1,4 \mathrm{GlcNAc}$ branches on the mannose core of $N$-glycans $(8,9)$. Many previous studies showed that GnT-IV 
is involved in the progression of cancers of the liver, pancreas, kidney and colon (10-13).

hCG is a glycoprotein hormone composed of $\alpha$ - and $\beta$-subunits. $\alpha$-subunit has two asparagine-linked ( $N$-linked) glycosylation sites and $\beta$-subunit has two $N$-linked and four serine-linked ( $O$-linked) glycosylation sites (14). Previous studies revealed that $\mathrm{N}$-glycans of hCG have many important biological functions (14-16) and that they are differentially modified in physiological and pathological states $(17,18)$. hCG from normal pregnant women and patients with hydatidiform mole contains only mono- and bi-antennary branched $N$-glycans. On the other hand, additional tri-antennary branched $N$-glycans are present on hCG of invasive mole and choriocarcinoma patients, and abnormal bi-antennary $\mathrm{N}$-glycan is detected on hCG from choriocarcinoma. The tri-antennary and abnormal bi-antennary branched $N$-glycans are biosynthesized by the action of GnT-IV on mono- and bi-antennary $N$-glycans. Takamatsu et al (19) confirmed the elevated activity of GnT-IV and higher expression level of GnT-IVa in choriocarcinoma cells compared to those in normal placentas, whereas the mRNA of $G n T-I V b$, another isoenzymatic form, was expressed at the same level in choriocarcinoma and placentas. Niimi et al (20) reported that GnT-IVa protein expression was strong in GTN (invasive mole and choriocarcinoma) and suggested that GnT-IVa might be involved in invasion of choriocarcinoma, using GnT-IVa suppression model. For further understanding of the role of GnT-IVa in choriocarcinoma, we established a choriocarcinoma cell line which overexpresses GnT-IVa and studied its target proteins for GnT-IVa glycosylation which contribute to the malignancy of choriocarcinoma.

\section{Materials and methods}

Tissue collection and processing. The present study was approved by the ethics committee of Nagoya University Graduate School of Medicine. Written informed consent was obtained before surgery from all patients with GTD for the use of clinical samples. Tissue samples from four cases of invasive mole and seven cases of choriocarcinoma were used for this study. Tissue samples were fixed in $10 \%$ formaldehyde, embedded in paraffin and routinely stained with haematoxylin and eosin for histological examination. All sections were histologically assessed by pathologists in our institute.

Cell lines and culture. Human choriocarcinoma cell line Jar was purchased from the American Type Culture Collection (ATCC; Manassas, VA, USA) and maintained in RPMI-1640 medium (Sigma-Aldrich, St. Louis, MO, USA) supplemented with $10 \%$ fetal bovine serum (FBS; Gibco, Grand Island, NY, USA), penicillin $(100 \mathrm{U} / \mathrm{ml})$, streptomycin $(100 \mu \mathrm{g} / \mathrm{ml})$, at $37^{\circ} \mathrm{C}$ in a humidified atmosphere of $5 \% \mathrm{CO}_{2}$ and $95 \%$ air.

Immunohistochemistry. Immunohistochemical staining for GnT-IVa protein was performed using a monoclonal antiGnT-IVa antibody (sc-100785; Santa Cruz Biotechnology, Inc., Santa Cruz, CA, USA) at a dilution of 1:400 as previously described $(20,21)$. Immunohistochemical staining for Griffonia simplicifolia lectin-II (GSL-II) was performed by a previous method with modification (22). Sections of $4 \mu \mathrm{m}$ thickness were deparaffinised, rehydrated and washed with phosphate-buffered saline (PBS). Endogenous peroxidase activity was blocked by incubating the sections in $0.3 \%$ hydrogen peroxide in $100 \%$ methanol. The sections were washed twice with PBS and once with $0.05 \mathrm{M}$ Tris-buffered saline (TBS). For antigen retrieval, the sections were treated with $0.1 \%$ trypsin solution in TBS containing $0.1 \% \mathrm{CaCl}_{2}$. Non-specific lectin-protein binding was blocked by incubating the sections in 5\% skim milk in PBS. The sections were then incubated overnight with biotinylated GSL-II (B-1215; Vector Laboratories, Inc., Burlingame, CA, USA) at a dilution of 1:400 in TBS containing $1 \%$ bovine serum albumin (BSA) and $10 \mathrm{mM} \mathrm{CaCl}{ }_{2}$ at $4^{\circ} \mathrm{C}$. After rinsing, the sections were immunostained using Vectastain Elite $\mathrm{ABC}$ kit (Vector Laboratories) for $30 \mathrm{~min}$ according to the manufacturer's instructions. 3,3'-diaminobenzidine tetrahydrochloride reagent (Nichirei, Corp., Tokyo, Japan) was used for colour development.

Construction of an overexpression vector of GnT-IVa and retroviral transduction. Construction of GnT-IVa-encoding retroviral vector was performed as previously described (23). Briefly, GnT-IVa was PCR amplified using primers (F, 5'-AA AAAGCAGGCTCCACCATGAGGCTCCGCAAT GG-3' and R, 5'-AGAAAGCTGGGTCAGTTGGTGGCTTTTTTAATA TGAATC-3') from the vector GnT-IVa/pSVL which was provided by Ogri et al (24). After confirming the sequence by subcloning the product into an entry vector, $G n T-I V a$ was transferred to the destination vector, pCLMSCV-puro-Rfa, using the Gateway system (Invitrogen-Life Technologies, Carlsbad, CA, USA). The retroviral vector of GnT-IVa (pCLMSCV-GnT-IVa/puro) was transfected into 293T cells with packaging vectors encoding viral gag-pol and envelope protein (pCL-GALV) as previously described (23), and the retroviral supernatant was transfected into Jar cells (Jar-GnT4a cells). Control vectors were transfected into Jar cells (mock cells) by the same methods. Jar-GnT4a and mock cells were selected by adding $1 \mu \mathrm{g} / \mathrm{ml}$ puromycin.

Western blot and lectin blot analysis. Whole cell lysate was prepared by lysing collected mock or Jar-GnT4a cells in Tris-NaCl-EDTA (TNE) buffer supplemented with protease inhibitor cocktail (Roche Diagnostics, Indianapolis, IN, USA). Secreted proteins from mock or Jar-GnT4a cells were obtained by concentrating the serum-free conditioned media in which they were cultured with Amicon ${ }^{\circledR}$ Ultra-4 Centrifugal Filter Units (Merck Millipore Ltd., Cork, Ireland). The protein concentrations were determined using BCA protein assay kit (Pierce, Rockford, IL, USA). Equal amounts of prepared proteins or immunoprecipitated proteins were separated by $10 \%$ sodium dodecyl sulphate-polyacrylamide gel electrophoresis (SDS-PAGE). Proteins on the gels were transferred onto polyvinylidene difluoride membranes. The membranes were blocked with 5\% skim milk in PBS, or 3\% BSA in TBS containing $0.05 \%$ Tween-20 (TBS-T) at room temperature for $1 \mathrm{~h}$, for western blot or lectin blot analysis, respectively. The membranes were incubated with a monoclonal antiGnT-IVa antibody (sc-100785, diluted 1:1,000; Santa Cruz Biotechnology), a monoclonal anti- $\beta$-actin antibody (A2228, diluted 1:1,000; Sigma-Aldrich), a polyclonal anti-integrin $\beta 1$ 
antibody (ab183666, diluted 1:5,000; Abcam plc., Cambridge, UK) and a monoclonal anti-lysosome-associated membrane protein-2 (LAMP-2) antibody (sc-18822, diluted 1:400; Santa Cruz Biotechnology) for western blot analyses, or biotinylated GSL-II (B-1215, diluted 1:1,000; Vector Laboratories) and Datura stramonium lectin (DSA) (B-1185, diluted 1:1,000; Vector Laboratories) for lectin blot analyses. The membranes were then incubated with horseradish peroxidase-conjugated anti-mouse IgG secondary antibody (\#7076S; Cell Signaling Technology, Inc., Danvers, MA, USA) or streptavidin-horseradish peroxidase (Vector Laboratories). Protein bands were visualized by developing the blots using ECL reagents (GE Healthcare, Buckinghamshire, UK) and ImageQuant LAS 4000 mini (GE Healthcare).

Cell proliferation assay. Cell proliferation was determined using modified tetrazolium salt (MTS) assay using the CellTiter $96^{\circledR}$ Aqueous One Solution Cell Proliferation assay (Promega, Madison, WI, USA). Cells $\left(5 \times 10^{3}\right)$ in $100 \mu 1$ of growth medium were plated in 96-well plates and incubated at $37^{\circ} \mathrm{C}$ for $72 \mathrm{~h}$. At 24,48 and $72 \mathrm{~h}, 20 \mu \mathrm{l}$ of MTS reagent was added to each well, followed by incubation for $2 \mathrm{~h}$ and measurement of absorbance at $490 \mathrm{~nm}$ by microplate reader (Multiskan Bichromatic; Labsystems, Helsinki, Finland).

Transwell migration and invasion assay. Migration assay and invasion assay were performed using the Transwell inserts (Corning Inc., Corning, NY, USA) with a filter of $6.5 \mathrm{~mm}$ diameter and $8 \mu \mathrm{m}$ pore size for 24-well plates as described earlier $(20,21)$. Invasion assay was performed using the upper chambers coated with Matrigel (Collaborative Biomedical Products, Bedford, MA, USA). Cells $\left(4 \times 10^{4}\right)$ in serum-free medium were plated in the upper chambers, while medium with $10 \%$ FBS was added to the lower chambers as chemoattractant. Cells on the upper side of the filters were scraped off after $24 \mathrm{~h}$ of incubation at $37^{\circ} \mathrm{C}$, and the filters were fixed and stained. The migration and invasion activities were evaluated by counting the number of migrated cells on the lower surface of the filters at X100 magnification.

Cell adhesion assay. Cell adhesion assay was performed as previously reported $(20,25)$. Cells $\left(4 \times 10^{4}\right)$ were plated in $100 \mathrm{ml}$ of medium in 96-well plates coated with fibronectin, collagen type I or collagen type IV (Becton-Dickinson, Franklin Lakes, NJ, USA). The plates were centrifuged at $200 \mathrm{x}$ g for $15 \mathrm{sec}$, and the cells were allowed to attach to each matrix at $37^{\circ} \mathrm{C}$ for $30 \mathrm{~min}$. After washing with PBS twice for eliminating non-adherent cells, absorbance at $492 \mathrm{~nm}\left(\mathrm{~A}_{492 \mathrm{~nm}}\right)$ was read using a microplate reader. The rate of cell adherence was calculated as follows: $\left[\mathrm{A}_{492 \mathrm{~nm}}\right.$ (matrix) - $\mathrm{A}_{492 \mathrm{~nm}}$ (no matrix)] $/ \mathrm{A}_{492 n m}$ (no matrix) (25).

Mouse studies. All animal procedures were reviewed and approved by the Nagoya University institutional Animal Experimentation Committee and performed in accordance with the institutional guidelines of Nagoya University Division of Experimental Animals. Five-week-old female nude mice (Balb/c slc nu/nu) were purchased from Japan SLC, Inc. (Shizuoka, Japan) and were injected subcutaneously with $5 \times 10^{5}$ mock or Jar-GnT4a cells in $200 \mu 1$ of growth medium $(\mathrm{n}=8$, each). Tumour size and health status of mice were monitored, and when the minor axis of tumours exceeded $5 \mathrm{~mm}$, tumour formation was considered to have occurred.

Immunoprecipitation. Whole cell lysate proteins or secreted proteins (200-1,000 $\mu \mathrm{g}$ ) from mock or Jar-GnT4a cells were incubated with antibodies $(10 \mu \mathrm{g})$ or biotinylated lectins $(80 \mu \mathrm{g})$ in TNE buffer containing protease protein inhibitor at $4^{\circ} \mathrm{C}$ overnight with rotary shaking. The immuno-complex was then incubated with resins at $4^{\circ} \mathrm{C}$ for $1 \mathrm{~h}$ with rotary shaking. Resins used were Protein G Sepharose 4 Fast Flow (GE Healthcare) for immunoprecipitation or Streptavidin Agarose Ultra Performance ${ }^{\mathrm{TM}}$ (Solulink Bioscience, Inc., San Diego, CA, USA) for lectin precipitation. The resins were used after equilibration with TBS-T. Resin bound immnune-complexes were washed five times with TBS-T and denatured by boiling at $100^{\circ} \mathrm{C}$ in SDS sample buffer.

Nano-LC/MS/MS. The immunoprecipitates were separated by SDS-PAGE and stained using Silver Stain MS kit (Wako Pure Chemical Industries, Ltd., Osaka, Japan). The protein bands were excised from the gels. Subsequent procedures and nano-LC/MS/MS analysis were conducted by Japan Proteomics (Sendai, Japan) using a standard protocol.

Flow cytometric analysis. For flow cytometric analysis, $1 \times 10^{5}$ cells were suspended in PBS containing $1 \%$ BSA and were incubated for $30 \mathrm{~min}$ with $0.2 \mu \mathrm{g}$ of a monoclonal antiLAMP-2 antibody (sc-18822; Santa Cruz Biotechnology). The cells were incubated with $2 \mu \mathrm{g}$ of an anti-mouse $\operatorname{IgG}(\mathrm{H}+\mathrm{L})$ antibody Alexa Fluor ${ }^{\circledR} 488$ conjugate (A-11029; Invitrogen/ Life Technologies) at $4^{\circ} \mathrm{C}$ for $30 \mathrm{~min}$. After washing with $3 \mathrm{ml}$ of PBS, flow cytometric analysis was performed using Attune ${ }^{\circledR}$ Acoustic Focusing Cytometer and Attune ${ }^{\circledR}$ Cytometric software (Applied Biosystems, Carlsbad, CA, USA). Mouse $\mathrm{IgG}_{1}$ isotype (MAB002; R\&D Systems, Inc., Minneapolis, MN, USA) was used for a negative control.

Statistical analysis. JMP PRO version 11.0.0 (SAS Institute, Inc., Cary, USA) was used for statistical analysis. Error bars in graphical data represent mean \pm standard deviation (SD). Differences were considered statistically significant at $\mathrm{P}<0.05$ in a two-tailed Student's t-test and log-rank test.

\section{Results}

Highly branched $N$-glycans present in invasive mole and choriocarcinoma. We first examined the expression of GnT-IVa and glycosylation level in invasive mole and choriocarcinoma by immunohistochemical staining of surgical specimens. GSL-II is a lectin which recognizes tri- or tetraantennary branched $N$-glycans modified by GnT-IVa (26). Immunostaining with GSL-II was strong in the invasive mole and choriocarcinoma and corresponded to that with anti-GnT-IVa antibody (Fig. 1). These results indicated that highly branched $N$-glycans modified by GnT-IVa are present in trophoblastic cells of invasive mole and choriocarcinoma.

GnT-IVa overexpression increases cell adhesion, migration and invasion in choriocarcinoma cells. To investigate the 

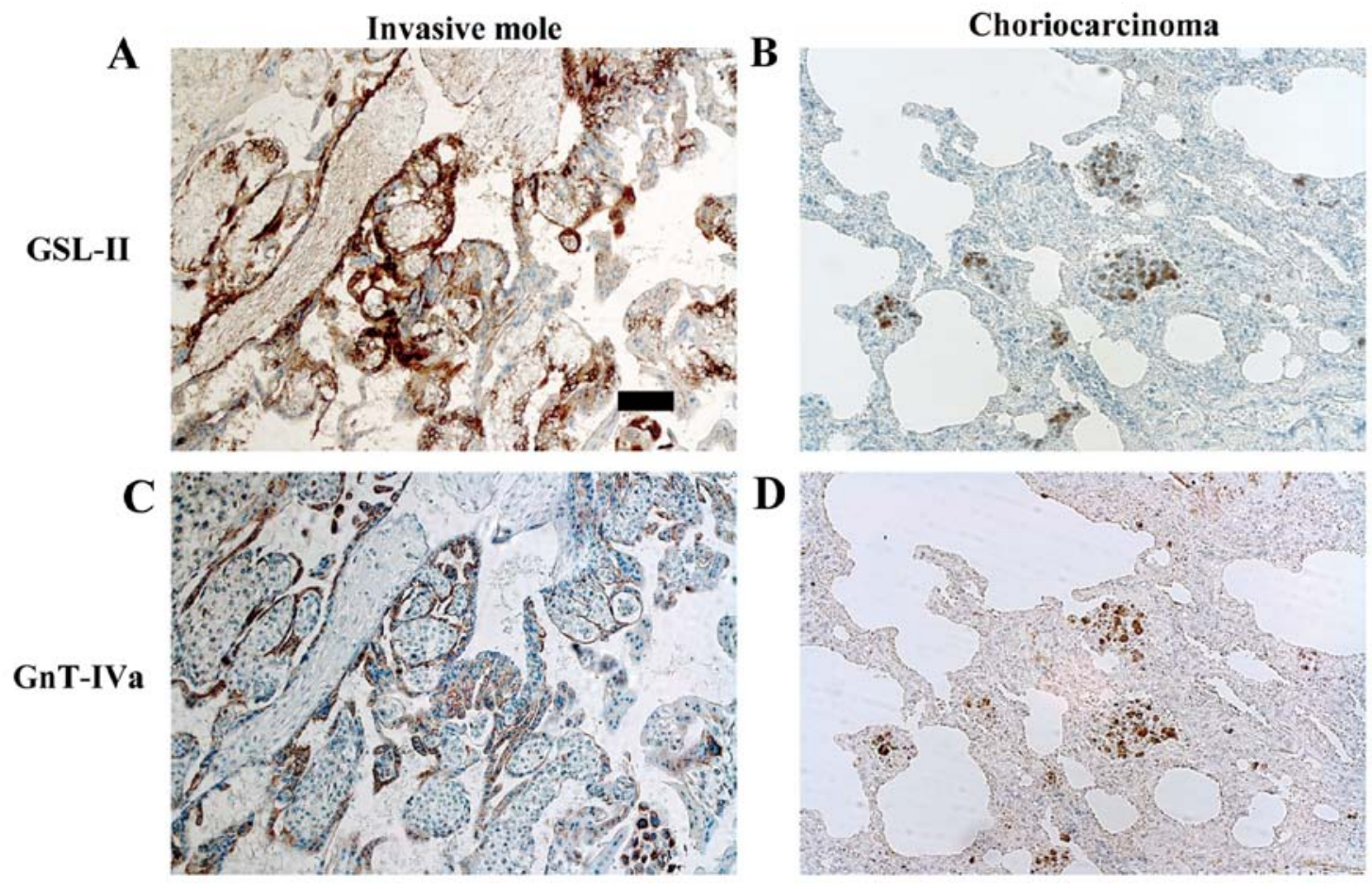

Figure 1. Highly branched $N$-glycans modified by GnT-IVa present in cells of invasive mole and choriocarcinoma. (A) Immunohistochemical staining using Griffonia simplicifolia lectin-II (GSL-II) in invasive mole and (B) choriocarcinoma. GSL-II is a lectin which recognizes tri- or tetra-antennary branched $\mathrm{N}$-glycans modified by GnT-IVa. (C) Immunohistochemistry using an anti-GnT-IVa antibody in invasive mole and (D) choriocarcinoma. Original magnification, $\mathrm{x} 100$; scale bar, $100 \mu \mathrm{m}$.

function of GnT-IVa in choriocarcinoma cells, we established a stable cell line that overexpressed GnT-IVa protein (Jar-GnT4a) using the choriocarcinoma cell line Jar. The expression of GnT-IVa protein was dramatically increased in Jar-GnT4a cells in comparison to that in parent Jar (WT) or transfectants with control gene (mock cells, Fig. 2A). We observed no significant difference between Jar-GnT4a cells and mock cells in cell proliferation for $72 \mathrm{~h}$, by MTS assay (Fig. 2B). The migration and invasion assay showed that the migratory and invasive abilities of Jar-GnT4a cells increased 2.5- and 1.4-fold, respectively, compared to those of mock cells (Fig. 2C). Next, we investigated the effect of GnT-IVa overexpression on cell adhesion to extracellular matrix (ECM). Cell adhesion assay showed that Jar-GnT4a cells had significantly greater adherence potential to ECM than that of mock cells (Fig. 2D). The number of Jar-GnT4a cells that adhered to fibronectin, collagen type I and collagen type IV were $2.88,2.86$ and 1.48 times higher, respectively, than that of mock cells.

In vivo effects of GnT-IVa overexpression on choriocarcinoma engraftment. We examined whether GnT-IVa overexpression increases malignant potential of choriocarcinoma cells in vivo. We subcutaneously injected Jar-GnT4a or mock cells into 5-week-old Balb/c slc nu/nu nude mice. The rate of tumour formation in mice injected with Jar-GnT4a cells was significantly higher than that in mice injected with mock cells $(\mathrm{P}=0.0407$; Fig. 2E). All of five mice and one of two mice that developed tumours injected with Jar-GnT4a cells and mock cells, respectively, died. The cumulative survival rate of mice with Jar-GnT4a cells was relatively lower (62.5 vs.
$12.5 \%$ ), although the difference was not statistically significant (Fig. 2F). Considered together, these results suggest that GnT-IVa overexpression promotes cell adhesion, migration and invasion of choriocarcinoma.

GnT-IVa overexpression increases highly branched $N$-glycans on integrin $\beta 1$. To investigate how GnT-IVa overexpression increased malignant potential of choriocarcinoma, we compared the protein expression patterns and the level of $\beta 1,4 \mathrm{GlcNAc}$ branches of $N$-glycans between Jar-GnT4a cells and mock cells. There was no significant difference in the protein levels of whole cell lysate and conditioned media between Jar-GnT4a and mock cells (left panels, Fig. 3A and B). However, lectin blot analyses with GSL-II and DSA which specifically recognize $\beta 1,4 \mathrm{GlcNAc}$ branches of $\mathrm{N}$-glycans, showed that the overexpression of GnT-IVa mediated the addition of $N$-glycans to several proteins in whole cell lysate or conditioned media (arrows in centre and right panels, Fig. 3A and B). Because GSL-II has high specificity for agalactosylated $\beta 1,4 \mathrm{GlcNAc}$ branches of $N$-glycans whereas DSA has high specificity for galactosylated $\beta 1,4 \mathrm{GlcNAc}$ branches of $N$-glycans, we used both lectins in these experiments.

Significantly greater adherence to ECM and higher rate of tumour formation in mice injected with Jar-GnT4a cells compared to those injected with mock cells suggested the possibility that an increase in highly branched $\mathrm{N}$-glycans on integrin $\beta 1$ might result from GnT-IVa overexpression. As expected, $\beta 1,4 \mathrm{GlcNAc}$ branches of $N$-glycans on integrin $\beta 1$ in whole cell lysate were increased, as illustrated by DSA blot analysis (Fig. 3C). These results suggest that GnT-IVa may 
A

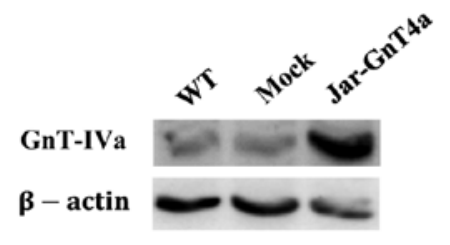

D

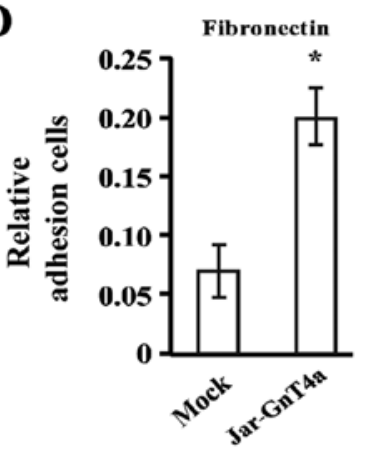

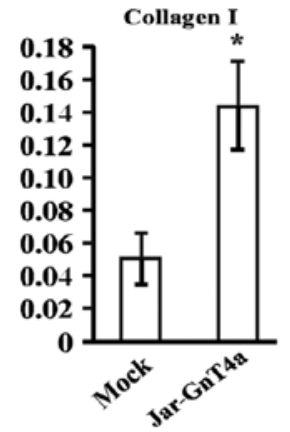

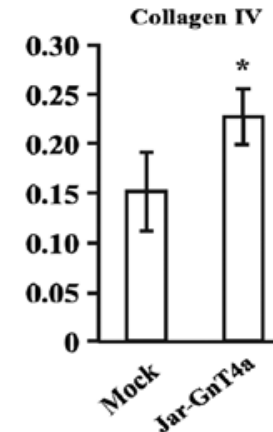

B

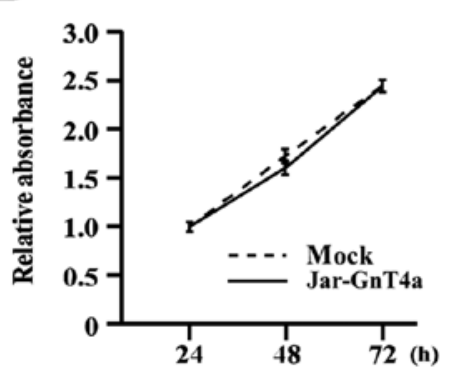

C

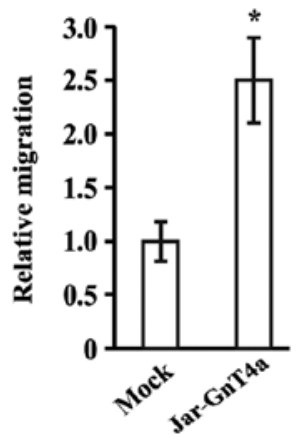

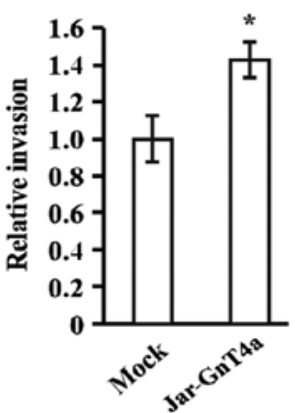

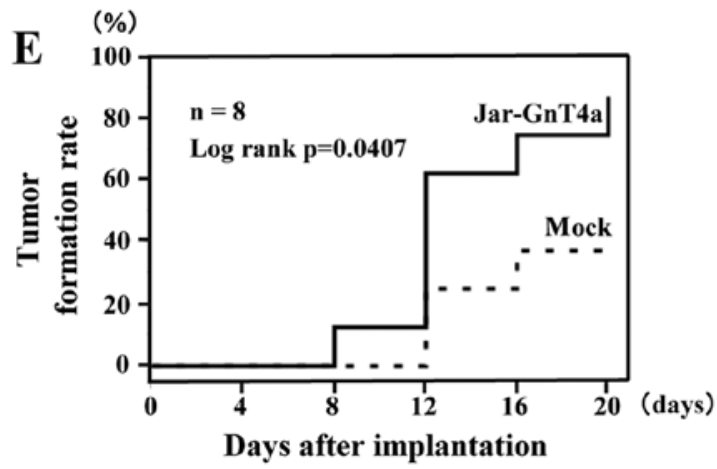

F

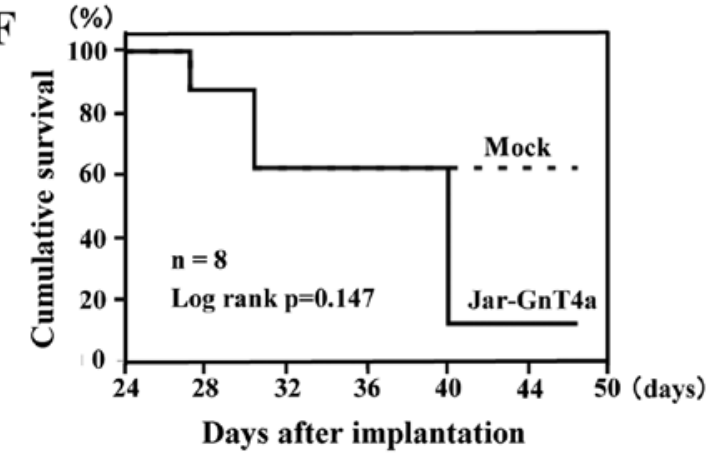

Figure 2. GnT-IVa overexpression increased cell adhesion, migration and invasion abilities of Jar cells. (A) GnT-IVa protein expression was increased in choriocarcinoma Jar cells transfected with GnT-IVa (Jar-GnT4a) compared with that in parent Jar cells (WT) or those transfected with control gene (mock). (B) Modified tetrazolium salt (MTS) assay showed that overexpression of GnT-IVa gene did not affect cell proliferation. The relative absorbance readings are depicted as mean \pm SD. Representative data are shown and three individual experiments yielded similar results. (C) Cell migration in Transwell migration assay and cell invasion in Matrigel invasion assay showing that GnT-IVa overexpression increased cell migration and invasion in Jar cells. Data were from experiments performed in triplicate and were normalized to those for mock cells. Each bar represents the mean \pm SD. "P<0.01. (D) GnT-IVa overexpression increased cellular interaction with ECM as demonstrated by the relative adhesion rates of mock cells and Jar-GnT4a cells on fibronectin, collagen type I and collagen type IV. Each bar represents the mean adherence rate \pm SD. ${ }^{*} \mathrm{P}<0.01$. Representative data are shown and three individual experiments yielded similar results. (E) The cumulative rate of tumour formation in mice injected with Jar-GnT-4a cells was significantly higher than that in mice injected with mock cells $(n=8$, each). (F) The cumulative survival rate of mice injected with Jar-GnT-4a was likely to be lower than that of mice injected with mock cells ( $\mathrm{n}=8$, each). WT, wild-type cells; mock cells, Jar cells transfected with control gene; Jar-GnT4a cells, Jar cells transfected with GnT-IVa.

contribute to the malignant behaviour of choriocarcinoma through decorating integrin $\beta 1$ with $N$-glycans.

LAMP-2 was one of the target proteins for $N$-glycan modification by GnT-IVa. To gain insight into the functional role of GnT-IVa in choriocarcinoma, we tried to find target proteins other than integrin $\beta 1$ for GnT-IVa glycosylation. We concentrated the target proteins in whole cell lysate and secreted proteins by lectin immunoprecipitation with DSA, because DSA blot analysis showed greater changes between Jar-GnT4a and mock cells than those by GSL-II blot analysis (Fig. 3A and B). DSA blot analyses and silver staining using immuno- precipitated proteins revealed that some proteins reacted more strongly in Jar-GnT4a cells than in mock cells (Fig. 4A and B). One and two protein bands containing separated whole cell lysate and conditioned media, respectively, were excised from the gels (arrowheads 1-3, Fig. 4A and B). Nano-LC/MS/MS analysis identified the proteins contained in the three bands (Table I). We focused on lysosome-associated membrane glycoprotein 2 (LAMP-2), because it was present in both whole cell lysate and conditioned media.

We examined whether highly branched $N$-glycans on LAMP-2 were increased by GnT-IVa overexpression. $\beta 1,4 \mathrm{GlcNAc}$ branches of $N$-glycans on LAMP-2 in whole cell 
A

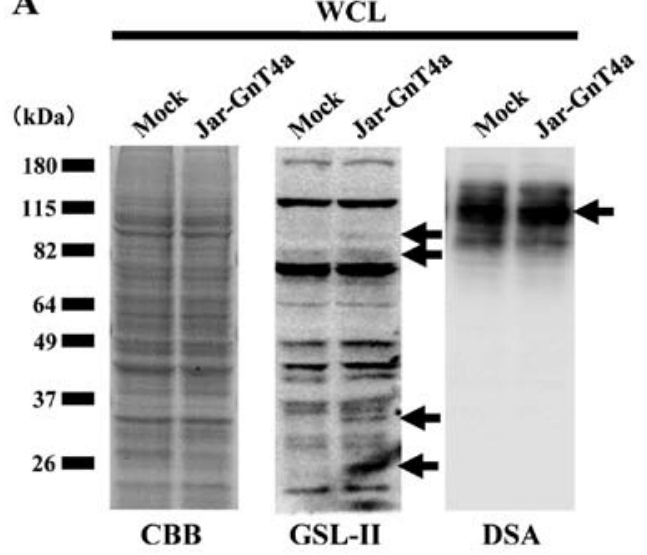

B

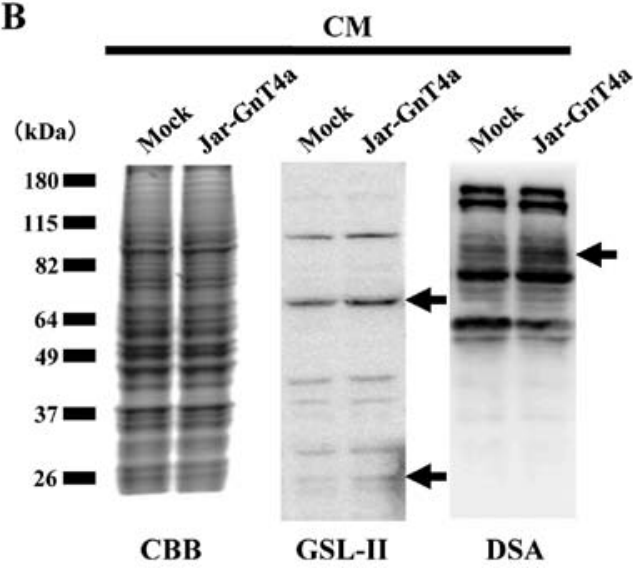

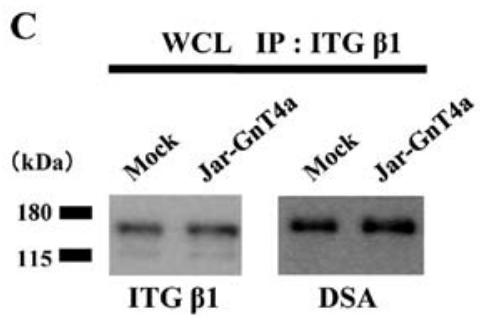

Figure 3. Highly branched $N$-glycans on integrin $\beta 1$ were increased by GnT-IVa overexpression. CBB staining, lectin blot analysis with GSL-II and DSA of (A) whole cell lysate proteins and (B) concentrated secreted proteins in conditioned media from mock or Jar-GnT4a cells. Protein bands which reacted more strongly with GSL-II or DSA in Jar-GnT4a cells than in mock cells, are indicated by arrows. (C) Whole cell lysate proteins from mock or Jar-GnT4a cells were immunoprecipitated by an anti-integrin $\beta 1$ antibody followed by western blot analysis with an anti-integrin $\beta 1$ antibody and DSA blot analyses. $\beta 1,4 \mathrm{GlcNAc}$ branches on integrin $\beta 1$ in Jar-GnT4a cells were increased compared to those in mock cells as demonstrated by DSA blot analysis, whereas the amount of integrin $\beta 1$ as measured by western blot analysis was not changed by GnT-IVa overexpression. WCL, whole cell lysate; CM, conditioned media; ITG $\beta 1$, integrin $\beta 1$.

Table I. Target proteins of GnT-IVa glycosylation in choriocarcinoma shown by Nano-LC/MS/MS analysis.

\begin{tabular}{llrr}
\hline Band no. & \multicolumn{1}{c}{ Protein } & Peptide matching & Protein coverage $(\%)$ \\
\hline 1 & Lysosome-associated membrane glycoprotein 2 & 4 & 11 \\
2 & $\alpha$ actin & 8 & 11 \\
2 & Lysosome-associated membrane glycoprotein 2 & 3 & 9 \\
3 & Phospholipid transfer protein & 1 & 3 \\
3 & Transferrin receptor protein 1 & 4 & 5 \\
\hline
\end{tabular}

The numbers of bands are the same in Fig. $4 \mathrm{~A}$ and $\mathrm{B}$.

lysate and conditioned medium were increased as illustrated by DSA blot analyses (Fig. 4C and D), indicating that LAMP-2 is one of the target proteins for GnT-IVa glycosylation and that it might contribute to the malignant potential of choriocarcinoma cells through GnT-IVa glycosylation. The differences in the reactivity of immunoprecipitated LAMP-2 between Jar-GnT4a and mock cells were relatively modest in DSA blot analyses in both whole cell lysate and conditioned media. This might be due to considerable expression of GnT-IVa in mock cells and LAMP-2 which carried $\beta 1,4$ GlcNAc branches of $N$-glycans may be substantially present in mock cells. The amount of LAMP-2 in whole cell lysate and conditioned media was confirmed by immunoprecipitation using an anti-LAMP-2 antibody. Next, we performed flow cytometric analysis to examine whether LAMP-2 was expressed on the cell surface.
Whole cell lysate contains cytoplasmic proteins as well as cell surface proteins. Our results showed that GnT-IVa overexpression changed the abilities of cell adhesion to ECM. Flow cytometric analysis revealed that LAMP-2 was present on the cell surface of Jar (Fig. 4E). Taken together, these results suggest that GnT-IVa may contribute to the malignant behaviour of choriocarcinoma through modifying $N$-glycans on integrin $\beta 1$ and LAMP-2 which is expressed on the cell surface.

\section{Discussion}

In the present study, we showed that GnT-IVa overexpression enhanced the malignant potential of choriocarcinoma in vitro and in vivo, by increasing cellular adhesion to ECM, 
A

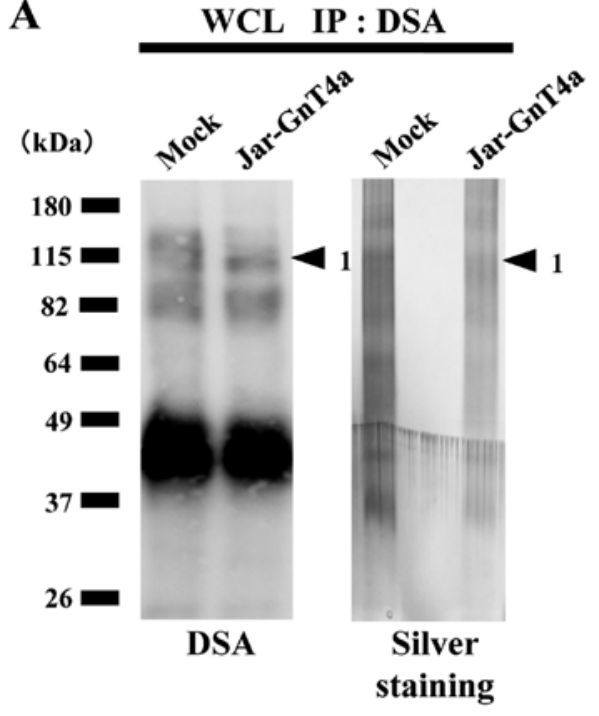

C

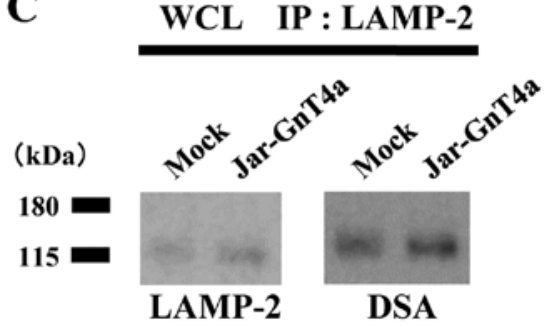

B

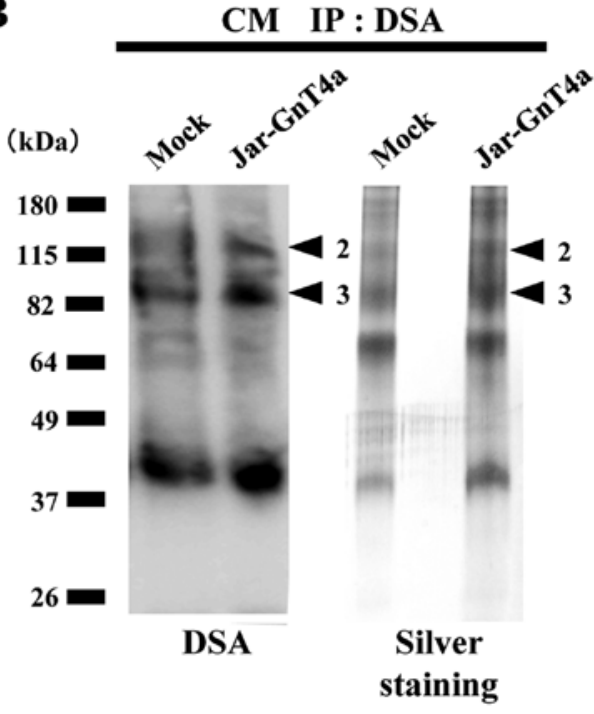

D

(kDa)

CM IP : LAMP-2

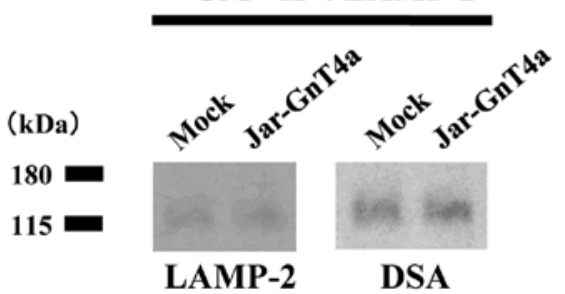

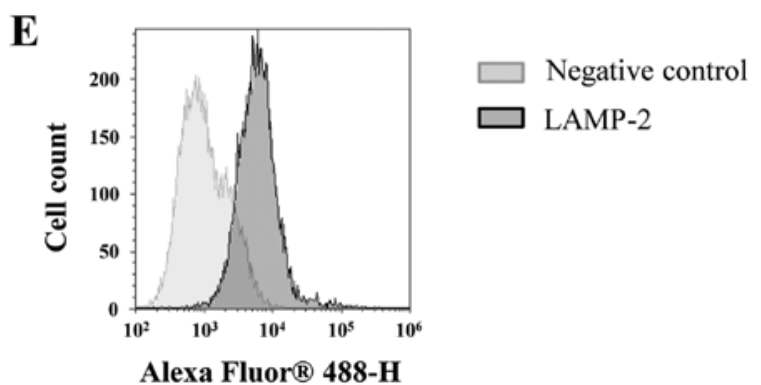

Figure 4. LAMP-2 was one of the target proteins for $N$-glycan modification by GnT-IVa. (A) Whole cell lysate proteins and (B) concentrated secreted proteins in conditioned media from mock or Jar-GnT4a cells were immunoprecipitated by DSA followed by DSA blot analysis and silver staining. Protein bands which reacted more strongly with DSA by GnT-IVa overexpression, are indicated by arrowheads with numbers 1-3. (C) Whole cell lysate proteins and (D) concentrated secreted proteins in conditioned media of mock or Jar-GnT4a cells were immunoprecipitated by an anti-lysosome-associated membrane glycoprotein 2 (LAMP-2) antibody followed by western blot analysis with an anti-LAMP-2 antibody and DSA blot analysis. $\beta 1$,4GlcNAc branches on LAMP-2 were increased in both whole cell lysate and conditioned media of Jar-GnT4a cells than those in mock cells, although the expression level of LAMP-2 was not changed by GnT-IVa overexpression. (E) Flow cytometric analysis showed that Jar cells expressed LAMP-2 on the surface of cell membranes. Three independent experiments yielded similar results.

cellular migration and invasion. These results complement the results of a previous study which showed that GnT-IVa knockdown in choriocarcinoma cells suppressed migration and invasion and decreased cellular adhesion to ECM (20). Immunohistochemistry using GSL-II demonstrated that highly branched $N$-glycans resulting from the action of GnT-IVa were strongly detected in invasive mole and choriocarcinoma, in proportion to the GnT-IVa protein expression. Therefore, GnT-IVa may play an important role in accelerating the malignancy of choriocarcinoma through addition of $\beta 1,4 \mathrm{GlcNAc}$ branches to the $\mathrm{N}$-glycans on some proteins. We tried to find the target proteins for GnT-IVa glycosylation.

Immunoprecipitation showed that integrin $\beta 1$ was a target protein of GnT-IVa glycosylation in choriocarcinoma.
Integrins are transmembrane cell adhesion molecules which function as cellular receptors of ECM ligands such as collagens, fibronectin and laminins (27). It has been reported that the $N$-glycan structure on integrin $\alpha$ or $\beta$ chains, resulting from the action of GnTs, affects integrin-mediated cell migration. Zhao et al (28) demonstrated that $\alpha 3 \beta 1$ integrin-mediated cell migration on laminin 5 was competitively regulated by GnT-III which catalyses the addition of bisected GlcNAc on integrin and suppresses cell migration. GnT-V which forms $\beta 1,6$ GlcNAc branched $N$-glycans enhances cell migration. In the present study, the results of cell adhesion assay and tumour formation in mice suggested that GnT-IVa may increase cell adhesion to ECM in choriocarcinoma, in vitro and in vivo. Niimi et al (20) reported that knocking down GnT-IVa in 
choriocarcinoma cells decreased $\beta 1,4 \mathrm{GlcNAc}$ branched $N$-glycans on integrin $\beta 1$ and suppressed integrin-mediated cell migration. These results support that GnT-IVa plays a role in the invasion of choriocarcinoma by increasing the $\beta 1,4 \mathrm{GlcNAc}$ branched $N$-glycans on integrin $\beta 1$.

Lectin immunoprecipitation and nano-LC/MS/MS revealed that LAMP-2 is another target protein of GnT-IVa glycosylation in choriocarcinoma. Some glycoproteins, such as integrin $\beta 1, \mathrm{CD} 147$, carcinoembryonic antigens, gammaglutamyl transpeptidases and glucose transporter-2 have been reported as target proteins for glycosylation by GnT-IVa in various carcinomas (11,29-31). LAMP-2 (CD107b) is a single-pass type 1 transmembrane protein which is normally present in the lysosomal membranes and occasionally on the surface of cell membranes in special situations including cancers (32-35). LAMP-2 is heavily glycosylated, to the extent that its carbohydrate side chains account for half of the molecular weight $(33,34)$. LAMP-2 has $16 \mathrm{~N}$-glycans which play crucial roles in cell adhesion to ECM and metastasis of cancer cells because $\mathrm{N}$-glycans configure cell surface ligands of cancer cells for galectin-1 (36), galectin-3 $(35,37)$ and selectins (38) on ECM or the membranes of the counter cell. Saitoh et al (38) reported that a colon carcinoma cell line with high metastatic potential expressed LAMP-2 at higher levels than that with low metastatic potential. In the present study, we demonstrated that LAMP-2 was expressed on the surface of choriocarcinoma cells and that the reactivity of DSA to $N$-glycans of LAMP-2 was increased by GnT-IVa overexpression. These results suggest that GnT-IVa may contribute to choriocarcinoma invasion by increasing $\beta 1,4 \mathrm{GlcNAc}$ branching on $N$-glycans of LAMP-2 as well as integrin $\beta 1$.

This study did not reveal the reason why LAMP-2 was detected in secreted proteins of Jar cells in this study, although it is very interesting that a transmembrane protein was present in conditioned media. We performed nano-LC/MS/MS on secreted proteins because the function of secreted proteins can be changed by glycosylation and affect cell invasion. It might be that a part of LAMP-2 on the cell surface was cleaved or included in exosomes and released into conditioned media which resulted in LAMP-2 detection in secreted proteins of Jar cells in this study. Previous studies reported that exosomal biomolecules (proteins, lipids, RNA and DNA) of cancer cells are associated with metastases to specific organs by preparing a pre-metastatic niche (39) and that $N$-glycosylation stabilizes LAMP-2 of exosomes and protects it from proteolysis for delivery of exosomes to target cells (40). LAMP-2 was detected in exosomes from HEK293FT cells which were transfected with tagged Lamp-2 introduction (40). It is suggested that cell membrane is included in the process of generating exosomes (41). It is possible that choriocarcinoma-specific tumor exosomes have LAMP-2 and promote organ-specific metastasis. However, more investigation is needed to explore the functional roles and localization of LAMP-2 in choriocarcinoma.

In conclusion, highly branched $N$-glycans resulting from the action of GnT-IVa were present in invasive mole and choriocarcinoma in proportion to GnT-IVa expression. GnT-IVa may facilitate invasion of choriocarcinoma by promoting cell adhesion, migration and invasion through $\beta 1,4$ GlcNAc glycosylation of integrin $\beta 1$ and LAMP-2.

\section{Acknowledgements}

We appreciate Dr Suguru Oguri (Tokyo University of Agriculture) and Dr Aruto Yoshida (Kirin Brewery Co.) for providing GnT-IVa/pSVL vector. We would like to thank Editage (www.editage.jp) for English language editing. The present study was supported by grant-in-aid no. 23592445 (to E.Y.) from the Japanese Ministry of Education, Culture, Sports, Science and Technology.

\section{References}

1. Berkowitz RS and Goldstein DP: Chorionic tumors. N Engl J Med 335: 1740-1748, 1996.

2. Lurain JR: Gestational trophoblastic disease I: Epidemiology, pathology, clinical presentation and diagnosis of gestational trophoblastic disease, and management of hydatidiform mole. Am J Obstet Gynecol 203: 531-539, 2010.

3. Lurain JR: Gestational trophoblastic disease II: Classification and management of gestational trophoblastic neoplasia. Am J Obstet Gynecol 204: 11-18, 2011.

4. Berkowitz RS and Goldstein DP: Current advances in the management of gestational trophoblastic disease. Gynecol Oncol 128: 3-5, 2013.

5. Varki A: Biological roles of oligosaccharides: All of the theories are correct. Glycobiology 3: 97-130, 1993.

6. Dennis JW, Laferté S, Waghorne C, Breitman ML and Kerbel RS: Beta 1-6 branching of Asn-linked oligosaccharides is directly associated with metastasis. Science 236: 582-585, 1987.

7. Pinho SS, Seruca R, Gärtner F, Yamaguchi Y, Gu J, Taniguchi N and Reis CA: Modulation of E-cadherin function and dysfunction by N-glycosylation. Cell Mol Life Sci 68: 1011-1020, 2011.

8. Oguri S, Minowa MT, Ihara Y, Taniguchi N, Ikenaga H and Takeuchi M: Purification and characterization of UDP- $N$-acetylglucosamine: $\alpha 1,3$-D-mannoside $\beta 1,4-N$-acetylglucosaminyltransferase ( $N$-acetylglucosaminyltransferase-IV) from bovine small intestine. J Biol Chem 272: 22721-22727, 1997.

9. Minowa MT, Oguri S, Yoshida A, Hara T, Iwamatsu A, Ikenaga $\mathrm{H}$ and Takeuchi $\mathrm{M}$ : cDNA cloning and expression of bovine UDP-N-acetylglucosamine: alpha1, 3-D-mannoside beta1,4- $N$-acetylglucosaminyltransferase IV. J Biol Chem 273: 11556-11562, 1998.

10. Ide Y, Miyoshi E, Nakagawa T, Gu J, Tanemura M, Nishida T, Ito $\mathrm{T}$, Yamamoto $\mathrm{H}$, Kozutsumi $\mathrm{Y}$ and Taniguchi $\mathrm{N}$ : Aberrant expression of $N$-acetylglucosaminyltransferase-IVa and IVb (GnT-IVa and b) in pancreatic cancer. Biochem Biophys Res Commun 341: 478-482, 2006.

11. Fan J, Wang S, Yu S, He J, Zheng W and Zhang J: $N$-acetylglucosaminyltransferase IVa regulates metastatic potential of mouse hepatocarcinoma cells through glycosylation of CD147. Glycoconj J 29: 323-334, 2012.

12. Zhu TY, Chen HL, Gu JX, Zhang YF, Zhang YK and Zhang RA: Changes in $N$-acetylglucosaminyltransferase III, IV and V in renal cell carcinoma. J Cancer Res Clin Oncol 123: 296-299, 1997.

13. D'Arrigo A, Belluco C, Ambrosi A, Digito M, Esposito G, Bertola A, Fabris M, Nofrate V, Mammano E, Leon A, et al: Metastatic transcriptional pattern revealed by gene expression profiling in primary colorectal carcinoma. Int $\mathrm{J}$ Cancer 115: 256-262, 2005.

14. Kobata A and Takeuchi M: Structure, pathology and function of the N-linked sugar chains of human chorionic gonadotropin. Biochim Biophys Acta 1455: 315-326, 1999.

15. Endo Y, Yamashita K, Tachibana Y, Tojo S and Kobata A: Structures of the asparagine-linked sugar chains of human chorionic gonadotropin. J Biochem 85: 669-679, 1979.

16. Kessler MJ, Reddy MS, Shah RH and Bahl OP: Structures of $\mathrm{N}$-glycosidic carbohydrate units of human chorionic gonadotropin. J Biol Chem 254: 7901-7908, 1979.

17. Mizuochi T, Nishimura R, Derappe C, Taniguchi T, Hamamoto T, Mochizuki M and Kobata A: Structures of the asparagine-linked sugar chains of human chorionic gonadotropin produced in choriocarcinoma. Appearance of triantennary sugar chains and unique biantennary sugar chains. J Biol Chem 258: 14126-14129, 1983. 
18. Mizuochi T, Nishimura R, Taniguchi T, Utsunomiya $\mathrm{T}$, Mochizuki M, Derappe C and Kobata A: Comparison of carbohydrate structure between human chorionic gonadotropin present in urine of patients with trophoblastic diseases and healthy individuals. Jpn J Cancer Res 76: 752-759, 1985.

19. Takamatsu S, Oguri S, Minowa MT, Yoshida A, Nakamura K, Takeuchi $M$ and Kobata A: Unusually high expression of $\mathrm{N}$-acetylglucosaminyltransferase-IVa in human choriocarcinoma cell lines: A possible enzymatic basis of the formation of abnormal biantennary sugar chain. Cancer Res 59: 3949-3953, 1999.

20. Niimi K, Yamamoto E, Fujiwara S, Shinjo K, Kotani T, Umezu T, Kajiyama H, Shibata K, Ino K and Kikkawa F: High expression of $N$-acetylglucosaminyltransferase IVa promotes invasion of choriocarcinoma. Br J Cancer 107: 1969-1977, 2012.

21. Yamamoto E, Ito T, Abe A, Sido F, Ino K, Itakura A, Mizutani S, Dovat S, Nomura $S$ and Kikkawa F: Ikaros is expressed in human extravillous trophoblasts and involved in their migration and invasion. Mol Hum Reprod 11: 825-831, 2005.

22. Suzuki O, Nozawa Y, Kawaguchi T and Abe M: Phaseolus vulgaris leukoagglutinating lectin-binding reactivity in human diffuse large B-cell lymphoma and its relevance to the patient's clinical outcome: Lectin histochemistry and lectin blot analysis. Pathol Int 49: 874-880, 1999.

23. Yamashita Y, Tsurumi T, Mori $\mathrm{N}$ and Kiyono $\mathrm{T}$ : Immortalization of Epstein-Barr virus-negative human B lymphocytes with minimal chromosomal instability. Pathol Int 56: 659-667, 2006.

24. Oguri S, Yoshida A, Minowa MT and Takeuchi M: Kinetic properties and substrate specificities of two recombinant human $N$-acetylglucosaminyltransferase-IV isozymes. Glycoconj J 23: 473-480, 2006

25. Yamamoto E, Ino K, Miyoshi E, Inamori $\mathrm{K}$, Abe $\mathrm{A}$ Sumigama S, Iwase A, Kajiyama $\mathrm{H}$, Shibata K, Nawa A, et al: $N$-acetylglucosaminyltransferase $\mathrm{V}$ regulates extravillous trophoblast invasion through glycosylation of alpha5beta1 integrin. Endocrinology 150: 990-999, 2009.

26. Nakamura-Tsuruta S, Kominami J, Kamei M, Koyama Y, Suzuki T, Isemura $\mathbf{M}$ and Hirabayashi J: Comparative analysis by frontal affinity chromatography of oligosaccharide specificity of GlcNAc-binding lectins, Griffonia simplicifolia lectin-II (GSL-II) and Boletopsis leucomelas lectin (BLL). J Biochem 140: 285-291, 2006

27. Desgrosellier JS and Cheresh DA: Integrins in cancer: Biological implications and therapeutic opportunities. Nat Rev Cancer 10 9-22, 2010

28. Zhao Y, Nakagawa T, Itoh S, Inamori K, Isaji T, Kariya Y, Kondo A, Miyoshi E, Miyazaki K, Kawasaki N, et al: $\mathrm{N}$-acetylglucosaminyltransferase III antagonizes the effect of $\mathrm{N}$-acetylglucosaminyltransferase $\mathrm{V}$ on alpha3beta1 integrinmediated cell migration. J Biol Chem 281: 32122-32130, 2006.
29. Yamashita K, Totani K, Kuroki M, Matsuoka Y, Ueda I and Kobata A: Structural studies of the carbohydrate moieties of carcinoembryonic antigens. Cancer Res 47: 3451-3459, 1987.

30. Yamashita K, Totani K, Iwaki Y, Takamisawa I, Tateishi N, Higashi T, Sakamoto Y and Kobata A: Comparative study of the sugar chains of gamma-glutamyltranspeptidases purified from human hepatocellular carcinoma and from human liver. J Biochem 105: 728-735, 1989.

31. Ohtsubo K: Targeted genetic inactivation of $\mathrm{N}$-acetylglucosaminyltransferase-IVa impairs insulin secretion from pancreatic beta cells and evokes type 2 diabetes. Methods Enzymol 479: 205-222, 2010.

32. Chen JW, Murphy TL, Willingham MC, Pastan I and August JT: Identification of two lysosomal membrane glycoproteins. J Cell Biol 101: 85-95, 1985.

33. Carlsson SR, Roth J, Piller F and Fukuda M: Isolation and characterization of human lysosomal membrane glycoproteins, h-lamp-1 and h-lamp-2. Major sialoglycoproteins carrying polylactosaminoglycan. J Biol Chem 263: 18911-18919, 1988.

34. Fukuda M, Viitala J, Matteson J and Carlsson SR: Cloning of cDNAs encoding human lysosomal membrane glycoproteins, h-lamp-1 and h-lamp-2. Comparison of their deduced amino acid sequences. J Biol Chem 263: 18920-18928, 1988.

35. Sarafian V, Jadot M, Foidart JM, Letesson JJ, Van den Brûle F, Castronovo V, Wattiaux R and Coninck SW: Expression of Lamp-1 and Lamp-2 and their interactions with galectin-3 in human tumor cells. Int J Cancer 75: 105-111, 1998.

36. Skrincosky DM, Allen HJ and Bernacki RJ: Galaptin-mediated adhesion of human ovarian carcinoma A121 cells and detection of cellular galaptin-binding glycoproteins. Cancer Res 53: 2667-2675, 1993.

37. Inohara $\mathrm{H}$ and Raz A: Identification of human melanoma cellular and secreted ligands for galectin-3. Biochem Biophys Res Commun 201: 1366-1375, 1994.

38. Saitoh O, Wang WC, Lotan R and Fukuda M: Differential glycosylation and cell surface expression of lysosomal membrane glycoproteins in sublines of a human colon cancer exhibiting distinct metastatic potentials. J Biol Chem 267: 5700-5711, 1992.

39. Hoshino A, Costa-Silva B, Shen TL, Rodrigues G, Hashimoto A, Tesic Mark M, Molina H, Kohsaka S, Di Giannatale A, Ceder S, et al: Tumour exosome integrins determine organotropic metastasis. Nature 527: 329-335, 2015.

40. Hung ME and Leonard JN: Stabilization of exosome-targeting peptides via engineered glycosylation. J Biol Chem 290: 8166-8172, 2015

41. Vanni I, Alama A, Grossi F, Dal Bello MG and Coco S: Exosomes: A new horizon in lung cancer. Drug Discov Today: Mar 10, 2017 (Epub ahead of print). doi: 10.1016/j.drudis.2017.03.004. 